\title{
Investigations on nano- and submicron-particle generation by spray painting processes
}

\author{
Q. Ye ${ }^{1^{*}}$, O. Tiedje ${ }^{1}$, S.R. Srinivas ${ }^{1}$, T. Noest ${ }^{2}$, U. Uhrner ${ }^{2^{*}}$ \\ ${ }^{1}$ Fraunhofer Institute for Manufacturing Engineering and Automation \\ Nobelstr. 12, 70569 Stuttgart, Germany \\ ${ }^{2}$ Institute of Internal Combustion Engines and Thermodynamics Graz University of Technology \\ Inffeldgasse 21a, 8010 Graz, Austria \\ *Corresponding author: qiaoyan.ye@ipa.fraunhofer.de and Uhrner@ivt.tugraz.at
}

\begin{abstract}
This paper presents experimental and numerical studies to determine the particle size distributions (PSD) and concentrations in paint overspray. Two kinds of paint materials, solvent borne and water borne paints, both with and without manufactured nanomaterials (pigments), and an industrial spray gun were used. Different aerosol measuring techniques, namely the Spraytec Fraunhofer type particle sizer for micro-sized droplets in the spray jet and the Scanning Mobility Particle Sizer (SMPS) for nano particles in paint overspray were applied. It was found, that solvent borne clear coats create significantly higher number concentration of nano-sized droplets than the water borneprimers. Only small differences in PSD between paints with and without manufactured nanomaterials were found. Numerical simulations of droplet trajectories within the spray booth, for both micro and nano sized droplets, were carried out. Based on the experimental and numerical results, a representative particle size distribution (smaller than $1 \mu \mathrm{m}$ ) for the given spray gun was obtained. Effects of turbulence models on the particle deposition on targets, especially for submicron particles, have been analysed in detailed.
\end{abstract}

\section{Keywords}

Nano- and submicron-particle, spray coating, aerosol measurement, droplet trajectory calculation

\section{Introduction}

The increasing application of manufactured nanomaterials (MNMs) in industrial products requires more knowledge about environmental and human safety. This is especially true for the spray painting process, which produces a high percentage of paint overspray. During the migration of the overspray droplets, solvent continues to evaporate, resulting in the formation of non-volatile fractions of paint aerosols that consist of paint matrix and the applied MNMs, such as $\mathrm{TiO}_{2}, \mathrm{ZnO}$, carbon black, etc. The MNMs could remain embedded in paint matrix particles or released from the paint matrix. Thus, the characterization of paint overspray aerosol becomes more and more important.

Information about the estimation of worker nanoparticle exposure to paint overspray is limited and particularly the process understanding of nanomaterial release during spray applications. A review of nanoparticle exposure at nanotechnology workplaces has been stated by Kuhlbusch et al. [1]. Carlton and Flynn [2] developed an empirical-conceptual model based on dimensional analysis, to predict breathing zone concentrations of a paint mist during spray painting tasks. Later, they also carried out CFD calculations to simulate production of breathingzone concentration of a paint overspray, for a simple case of the spray painting of a flat plate in a cross-flow ventilated booth [3].

For the risk estimation of exposure to paint overspray, it is also useful to categorize the paint aerosol particles in a spray jet, which can provide important information with regard to the worst case for a given spray atomizer and the applied paint material. Based on the experimental and numerical studies [4-6], paint droplet injection models for different atomizers and corresponding application parameters have been developed, with which the spray transfer efficiency TE (amount of paint reaching the work piece) as well as the paint overspray can be well predicted. However, these studies considered particle dispersion and deposition mainly for droplets larger than $1 \mu \mathrm{m}$. Study on the generation and characterization of nano- and sub-micro paint aerosols for a given atomizer is still quite scarce.

Recently Göhler and Stintz [7] analysed the airborne particle release from spray guns by sampling small particles in a turbulent pipe flow. The results showed that depending upon the material and pray unit used, $5 \times 10^{8}$ to $3 \times 10^{8}$ particles were released per gram of paint ejection, of which around $10 \mathrm{no} \%$ to $60 \mathrm{no} \%$ were finer than $100 \mathrm{~nm}$. These results were later applied to their CFD-modelling of nano particle dispersion for the prediction of worker exposure [8]. 
Concerning to the particle transport in turbulence flows, especially for nano particles, there are many reports [912], in which the effect of different turbulence models, wall treatments and models of turbulent dispersion on particles were investigated using CFD tools. In many of such studies, direct numerical simulation (DNS) data of channel flows were applied to create damping functions for predicting more accurate particle fluctuation velocity in near wall turbulent flow. However, in spray painting applications the spray jet is characterized with turbulent impinging jet. There is no available DNS data for such complicated turbulent near wall flow with high Reynolds number.

Currently, a joint transnational research project (NanoGeCo) has been set up, focusing on detailed characterization of nanoparticles by atomization processes in spray painting. Within this project different stages of experimental and numerical studies have been identified. In this stage of the research, determination of particle size distributions and concentrations in paint overspray were carried out. Two kinds of paint materials, solvent borne and water borne paints, both with and without manufactured nanomaterials (pigments), and an industrial spray gun were used. Different aerosol measuring techniques, namely the Spraytec Fraunhofer type particle sizer for micro-sized droplets in the spray jet and the Scanning Mobility Particle Sizer (SMPS) for nano particles in paint overspray were applied.

Numerical simulations of droplet trajectories within the spray booth for both micro and nano sized droplets were carried out, taking into account, the solvent evaporation in the droplets. Droplet solid fractions in overspray and droplet transfer efficiencies on a target were analysed, especially for nano- and submicron droplets. The effects of turbulence models on the dispersion and deposition of small sized particles were studied using RANS models (Renolds Averaged Navier-Stokes), namely k- $\varepsilon$ and RSM (Reynolds stress model). The simulated number concentrations of nano-sized droplets were compared with the measurements, resulting in an improved injection model for nano sized droplets. Based on the experimental and numerical results, a representative particle size distribution (smaller than $1 \mu \mathrm{m}$ ) for the given spray gun has been obtained, which provides information for further investigations, to assess human exposure to nano particles, in a practical paint booth and for toxicological studies of atomized nano-particles.

\section{Experimental study}

Paint materials, spray gun and spray booth

All measurements were carried out in a practically relevant painting booth with a well-defined homogeneous booth air velocity of $0.3 \mathrm{~m} / \mathrm{s}$. A HVLP-spray gun (High Volume Low Pressure) from SATA was used. A flat plate with size of $200 \times 800 \mathrm{~mm}^{2}$ as droplet deposition target was located horizontally. Painting distance between the gun and the target was $180 \mathrm{~mm}$. Table 1 shows the application parameters. Table 2 summarizes the properties of used paint liquids.

Table 1: Operating parameters

\begin{tabular}{|l|l|}
\hline Liquid flow rate & $150[\mathrm{~g} / \mathrm{min}]$ \\
\hline Gun air flow & $410[\mathrm{NL} / \mathrm{min}]$ \\
\hline Gun pressure & $2[\mathrm{bar}]$ \\
\hline Painting distance & $180 \mathrm{~mm}$ \\
\hline
\end{tabular}

Table 2: Properties of paint materials

\begin{tabular}{|l|l|l|l|}
\hline Name & $\begin{array}{l}\text { Density (wet paint) } \\
\mathrm{kg} / \mathrm{m}^{3}\end{array}$ & $\begin{array}{l}\text { Density (dry) } \\
\mathrm{kg} / \mathrm{m}^{3}\end{array}$ & $\begin{array}{l}\text { Non-volatile } \\
\text { fraction }\end{array}$ \\
\hline Clear coat & 999.2 & 1160 & $53.1 \%$ \\
\hline Primer & 999.0 & 1135 & $13.3 \%$ \\
\hline
\end{tabular}

Basically, two-component, solvent borne clear coat and water borne paint (primer) were used. Paint materials with and without pigments ( $\mathrm{ZnO}$ and carbon black) were applied in measurements. A nanoparticle concentration of approximately $1.5 \mathrm{v} / \mathrm{v} \%$ calculated on solid binder was used, corresponding to the normal coating applications. It was found that there were no significant effects of pigments or MNM on the listed properties of paint liquids and as well as on the rheological behaviours, as shown in Fig. 1. Both liquids shows shear thinning behaviour of viscosity, but it is stronger for the primer. However, the apparent viscosity of the clear coat is significantly lower than that of the primer. The viscosity curves in Fig. 1 can be helpful for understanding the measured droplet size distributions shown later. For clear coat the solvent is composed mainly of Butyl acetate that was used in the droplet trajectory calculation in the numerical study.

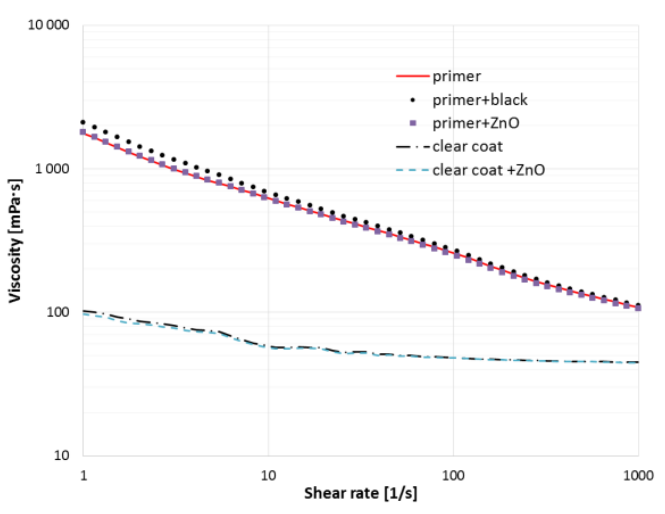

Figure 1. Viscosity measurement (shear curve) of the paint materials 
Micro droplet size measurement in spray jet

In terms of mass, the spray jet air flow is mainly influenced by micro-sized particles and to a far lesser extent by nanoparticles. It is, therefore, necessary to measure micro droplet size distribution in a spray jet, in order to correctly model spray jet turbulent flow using CFD tools. Figure 2 shows the droplet size measurement setup using a Malvern Spraytec Fraunhofer type particle sizer. A distance of $z=50 \mathrm{~mm}$ from the nozzle was chosen and the laser beam of the Malvern Spraytec was oriented along the $x$ axis. The spray gun that was mounted on a robot was traversed along the major axis y of the elliptical spray cone. A similar approach was applied in our previous studies $[5,6]$.

Based on the individual droplet size distributions in the elliptical spray region, the integral distribution of the whole spray region was then calculated and shown in Fig. 3. The corresponding Sauter mean diameter $D_{3,2}$ is $7.11 \mu \mathrm{m}$ for the primer and $6.52 \mu \mathrm{m}$ for the clear coat. In order to show more clearly the difference in size distributions for small particles between the two paint liquids, mean diameter Dv10 (10 percent of particle volume accumulation distribution lies below the Dv10, which characterizes the small particle size) along the elliptical spray cone are depicted in Fig. 4. Clearly, the clear coat creates somewhat finer particles (Dv10) than the primer. Measurements of nanoparticle size distributions created from the paint liquids will be illustrated in the next section.

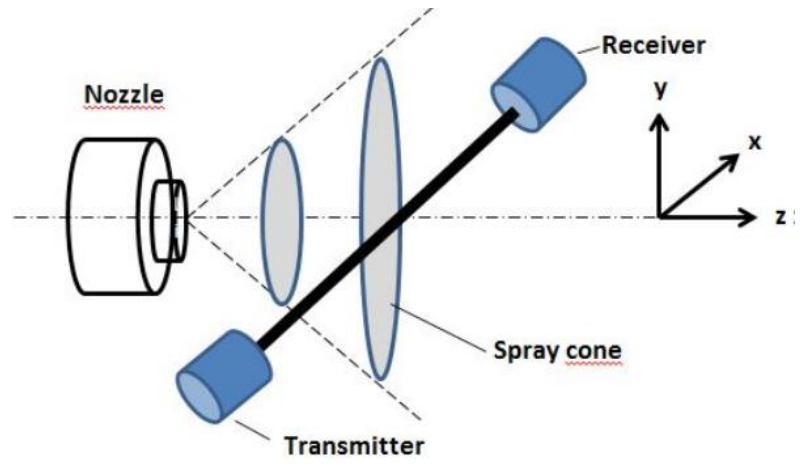

Figure 2. Schematic of the droplet size measurement setup

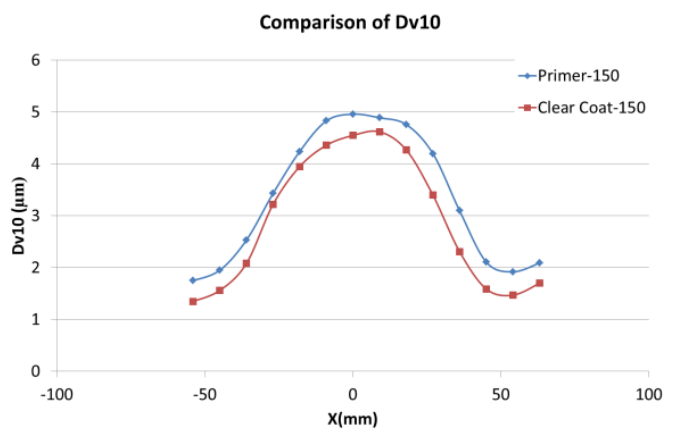

Figure 4. Mean particle size Dv10 distributions along the spray cone

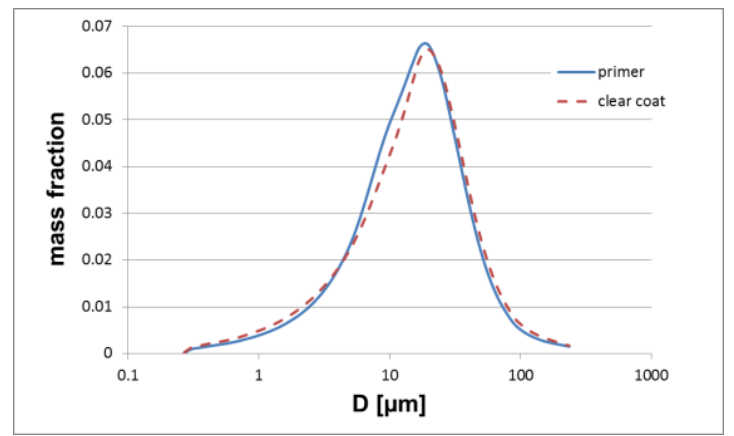

Figure 3. Integral droplet size distributions

\section{Nano and submicron particle concentration in spray overspray}

Particle size distribution (PSD) measurements in the nanometre range require on one hand a certain time interval scanning from a few nanometres to several hundreds of nanometres including an instrument specific reset time (retrace time). Therefore, a varying evaporation speed of droplets in overspray as well as coagulation of droplets and further atomization and dilution processes at the same time makes a representative sampling difficult. The paint aerosol is sticky, which may make the sensor signal quickly messy during data sampling close to overspray with high concentration. With this consideration an experimental arrangement and setup was established, as shown in figure 5. Basically, a target plate was used for depositing large paint droplets. Two measuring points $(\mathrm{L} 50 \mathrm{~cm}$ and $\mathrm{S} 30 \mathrm{~cm})$ were located in the middle of the two plate edges, but far away from the edges and at the same level of the plate $(\mathrm{H} 79.5 \mathrm{~cm})$. The quasi-static spray jet flow allows subsequent scans during atomization 
and facilitates repeatability of measurements. A SMPS (scanning mobility particle sizer) and a CPC (condensation particle counter) were applied to measure nanoparticles of paint overspray. The SMPS/CPC was operated in "batch" mode. An impactor is used as a pre-filter to limit the measurement range to sub-micron particles and to avoid damage due to overflow to the instruments sensitive parts. Cleaning on the impactor had to be carried out frequently, in order to obtain accurate results. Parameters such as sheath air flow and scanning time were set to 3 $1 /$ min and 60 seconds.

Background particle number concentration (PNC) in the spray booth with the booth and gun air flow was always measured before each operation of spray gun and is shown in Figs. $6-8$. In Fig. 6 results are shown for primer and primer with pigments (carbon black). For the water borne paint (Fig. 6) it can be observed that nanoparticle concentrations are higher than those of the background for particles with mobility diameter $D_{p}>200 \mathrm{~nm}$. It can be also seen that number distribution for the primer with carbon black is a little bit lower than that of primer.

Figures 7 to 8 shows results from solvent borne paints (clear coat with and without $\mathrm{ZnO}$ ) for the measuring positions at $L=50 \mathrm{~cm}$ and $S=30 \mathrm{~cm}$. The PNC differences between paint aerosols and the background are generally small in the $40 \mathrm{~nm}$ to $80 \mathrm{~nm}$ range. The difference between clear coat with and without $\mathrm{ZnO}$ is also small. Significant higher PNC can be observed for particles in the range of 100 to $640 \mathrm{~nm}$. The PNC at measuring position $\mathrm{L}=50 \mathrm{~cm}$ is higher than that at $S=30 \mathrm{~cm}$, which delivers important information for the nanoparticle dispersion for the trajectory modelling in the numerical study. Comparing the two paints, PNCs of primer are during the atomization process far lower than those of the solvent borne paints. Primers showed a very "stick character" and we assume that these differences are due to the high viscosity of these paints.

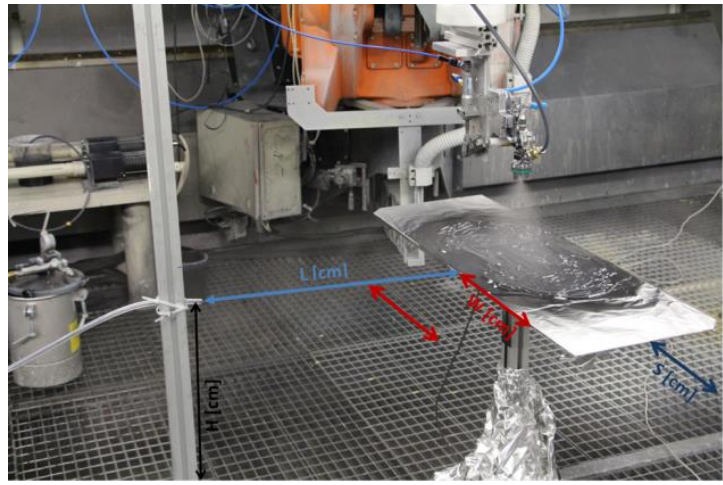

Figure 5. Setup of the spray application and sampling position definition.

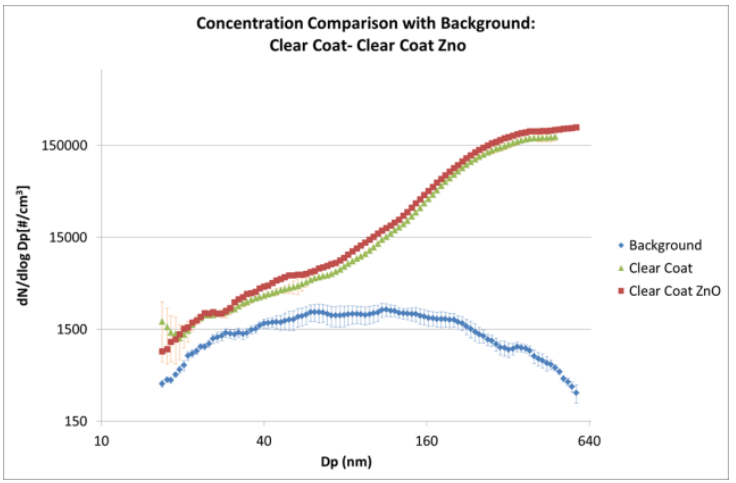

Figure 7. Clear coat particle concentration at the measuring position: $\mathrm{L} 50 \mathrm{~cm}, \mathrm{~W} 0, \mathrm{H} 79.5 \mathrm{~cm}$

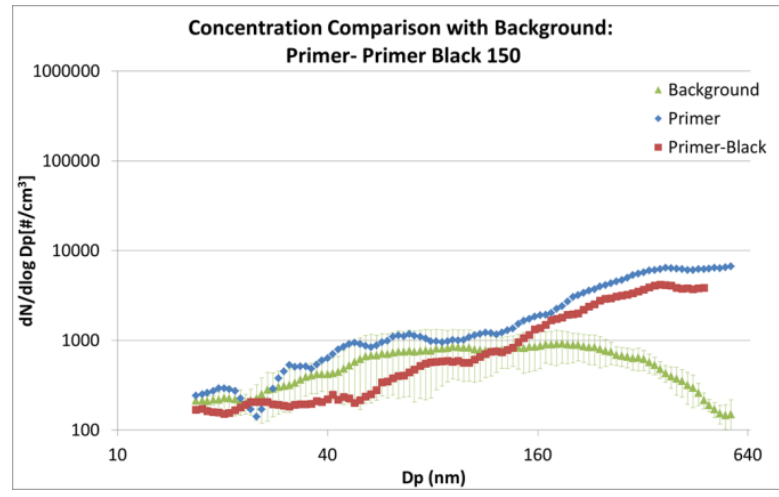

Figure 6. Primer particle concentrations at the measuring position: $\mathrm{L} 50 \mathrm{~cm}$, WO $\mathrm{H} 79.5 \mathrm{~cm}$

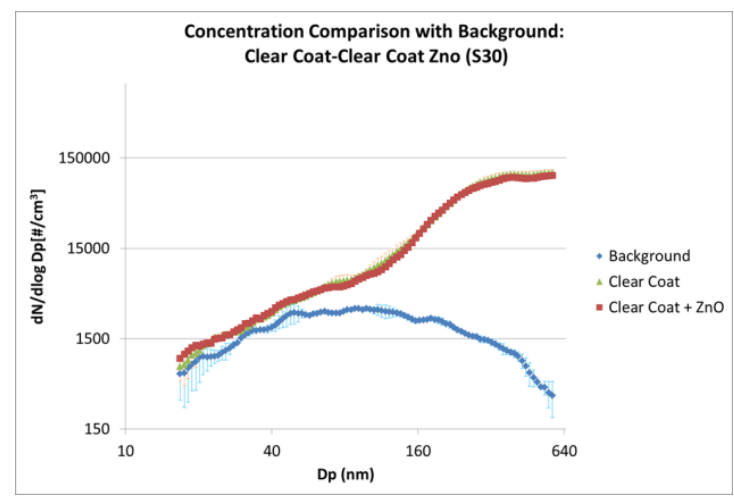

Figure 8. Clear coat particle concentration at the measuring position: $\mathrm{S} 30 \mathrm{~cm}, \mathrm{H} 79.5 \mathrm{~cm}$

\section{Numerical study}

Numerical methods

The commercial CFD code ANSYS-Fluent 17, based on the finite-volume approach, was used for the numerical simulations. The gas phase was modelled using the Eulerian conservation equations of mass, momentum, and energy. The three-dimensional compressible airflow was directly simulated from the nozzle using the coupled solver, as it was found to be more stable than the segregated solver for this specific airflow calculation. As inlet 
boundary conditions, booth air velocity of $0.3 \mathrm{~m} / \mathrm{s}$ and air mass flow rate and stagnation temperature were used at the air nozzles.

An unstructured mesh with 3.3 million cells was used to discretize the computational domain of $3.5 \times 2.5 \times 2.0 \mathrm{~m}^{3}$, including the complicated spray gun geometry. Mesh refinement was carried out. Prism mesh layers on the target wall were created with the first layer thickness of $0.5 \mathrm{~mm}$. For this mesh model the turbulence Realizable k- $\varepsilon$ model with scalable wall function was applied, since the dimensionless wall distance $\mathrm{y}^{+}$ranges from $1-20$ on the plate.

It is well known that the particle deposition is strongly influenced by the used near wall turbulence models, especially for small size particles. An isotropic turbulent flow is basically assumed in two equation RANS model (Reynolds Averaged Navier-Stokes), which results in an over estimation of particle deposition rate if the original eddy interaction model (EIM) in common CFD-codes is used [10-12]. In order to model the near-wall anisotropy flow using k- $\varepsilon$ models, some modifications [9-10] of EIM have been performed previously by using damping functions that were derived using DNS-data of channel flows. However, such approach is not suitable to the present complicated turbulent impinging jet. Instead of using damping functions the Reynold-stress turbulence model (RSM), which can account for anisotropic turbulence, could be applied to particularly study dispersion and deposition of quite small particles. However, the RSM is quite sensitive to the grid quality. The present simulations with RSM using tetrahedral mesh near the atomizer resulted in an unrealistic spray jet. For this reason a computational domain with hexahedral meshes but without atomizer was used to study the near wall nanoparticle deposition and dispersion. The obtained air flow and the particle trajectories in the near-atomizer region based on the $\mathrm{k}-\varepsilon$ model were exported then for the inlet conditions in the hexahedral mesh model. Reasonable results could be obtained, as shown later.

Droplet trajectories were calculated using the Lagrangian particle tracking method (LPT) by integration of the equation of motion,

$\frac{d \mathbf{u}_{\mathrm{p}}}{d t}=f_{D}\left(\mathbf{u}-\mathbf{u}_{\mathrm{p}}\right)+\mathbf{F}_{G}+\mathbf{F}_{L}, \quad \frac{d \mathbf{x}}{d t}=\mathbf{u}_{\mathrm{p}}$,

in which the drag force $f_{D}\left(\mathbf{u}-\mathbf{u}_{\mathrm{p}}\right)$ and the gravity force $\mathbf{F}_{G}$ (force/unit particle mass) are dominant forces for large droplets. For nano- and sub-micro droplets the Saffman's lift force $\mathbf{F}_{L}$ and the Stokes-Cunningham drag laws were taken into account. The stochastic tracking model with random walk and eddy lifetime was applied to calculate the instantaneous air velocity $\mathbf{u}$. For $\mathrm{k}-\varepsilon$ model the fluctuating velocity component is equal to $(2 \mathrm{k} / 3)^{1 / 2}(\mathrm{k}$ is turbulent kinetic energy), whereas anisotropy fluctuating components: (uu $)^{1 / 2}$, (vv) ${ }^{1 / 2}$ and (ww) ${ }^{1 / 2}$ can be obtained using RSM. The other important issue for modelling the turbulence dispersion on the droplet motion is the determination of integral time scale constant that is used for calculation of the particle-eddy interaction time in the EIM,

$T_{L}=C_{L} \frac{k}{\varepsilon}$

In Fluent the integral time scale constant $C_{L}$ is 0.15 by default, which can be modified for the trajectory calculation of small particles as suggested in previous works $[9,16]$. A Larger value $C_{L}=0.8$ was used in current study, in order to achieve better agreement with experimental results of the nanoparticle dispersion. The corresponding effect of $C_{L}$ is shown in the following section.

Droplet size distributions shown in Fig. 3 were used to create injection data for the trajectory calculation of microsized particles. The injection position was located quite close to the atomizer. The initial droplet injection velocities were fitted by using the information from the gas flow field and by matching the film thickness distribution on the flat plate. The detailed approach for creating injection data can be referred to in our previous studies [5, 6]. Water and Butyl acetate were used as solvents in calculation of droplet evaporation for the water borne coat and the clear coat respectively. Species transports in gas phase, such as air- Butyl acetate and air-water-steam, have been performed. Raoult's law was used to calculate the pressure equilibrium at the gas/liquid interface. Detailed physical models are shown in reports $[14,15]$. Two phase coupling was taken into account.

Static film thickness distribution, namely the film growth rate $[\mu \mathrm{m} / \mathrm{s}]$ on the target can be obtained after particle trajectory calculation. The spray gun is static in the present numerical simulation, hence in order to compare with the measured dynamic film thickness distribution, the simulated static film pattern has to be integrated, taking into consideration the robot velocity, the wet as well as the dry density of the paint material. Finally, the simulated two phase bulk flow field was applied to study the dispersion/deposition of nano- and sub-micro particles. 


\section{Simulation results}

Trajectory calculation for spray jet using micro-meter sized particles

At first, air flow using $k-\varepsilon$ turbulence model and trajectory calculations using micro-sized particles were carried out. Figure 9 shows the air velocity close to the atomizer. High velocities about $370 \mathrm{~m} / \mathrm{s}$, can be observed at the air nozzles. Droplets were injected in a circular region with a radius of $2 \mathrm{~mm}, 5 \mathrm{~mm}$ downstream the liquid nozzle and above the cross-section of the shaping air flow jets. The flat spray jet, as shown in Fig. 10, was created because of the shaping air flow jets. To provide a sensible resolution of the entire flow field, the velocity contours are depicted in the range of $0-30 \mathrm{~m} / \mathrm{s}$ in Fig. 10. A quite narrow elliptic flow region is formed with a narrow extension along $z$, which results in a narrow elliptic film pattern on the plate (the so-called static film thickness distribution or static film growth rate $[\mu \mathrm{m} / \mathrm{s}])$. The calculated dynamic dry film thickness profile was compared with the experimental result that was measured by means of magneto-inductive method. A good agreement between measured and predicted film thickness was obtained (Fig. 11). Except the film thickness distribution, non-volatile fractions in droplets for both paint liquids were also analysed. As shown in Fig. 12, solvents were fully evaporated for droplets with diameter smaller than $1 \mu \mathrm{m}$, which delivers important information for the nanoparticle sampling measurement. A re-condensation in paint overspray can be well neglected, since the solvent concentration far away from the spray jet is quite low and the process temperature is constant. The validated numerical simulation results in this section provide the reasonable flow field of spray jet for the further study of dispersion/deposition of nanoparticles.

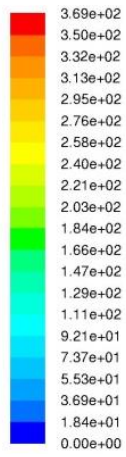

Figure 9. Velocity contours near the atomizer

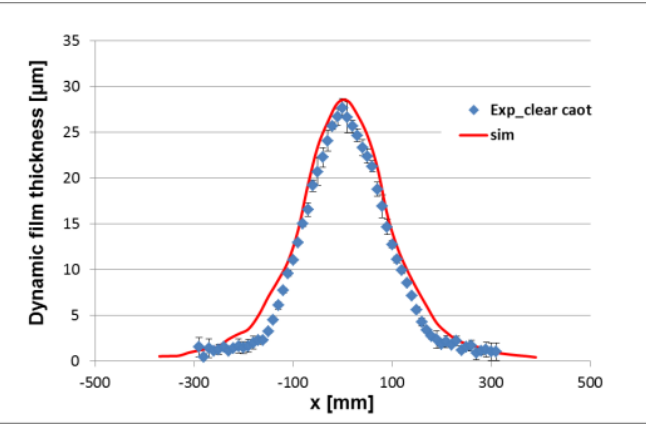

Figure 11. Comparison of measured and calculated dynamic film thickness distributions for clear coat

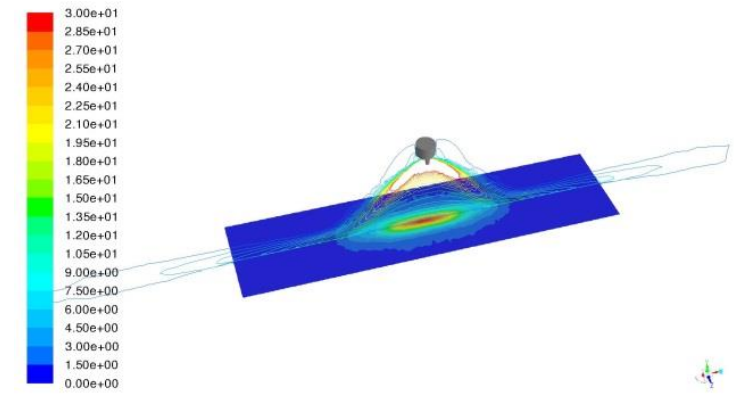

Figure 10. Calculated velocity contours colored by velocity magnitude $[\mathrm{m} / \mathrm{s}]$ in the plane $\mathrm{z}=0$. The static film thickness distribution on the plate is also overlaid.

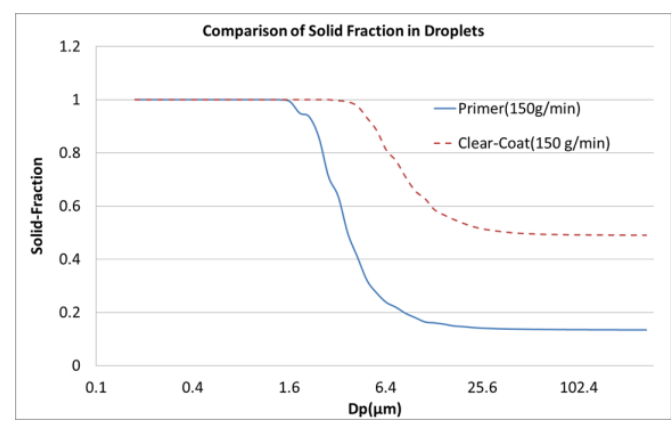

Figure 12. Comparison of solid (non-volatile) fraction in droplets on the target plate

\section{Trajectory calculation with consideration of nanoparticles}

As mentioned above, a computational domain $\left(3.5 \times 0.22 \times 2.0 \mathrm{~m}^{3}\right)$ with a hexahedral mesh model without atomizer was used so that simulations of turbulent flow with RSM could be carried out without any difficulty. In order to study effects of turbulence models on particle deposition, simulation with the k- $\varepsilon$ model was also performed. The air flow field and particle trajectory information downstream from the liquid nozzle in Fig. 10, namely at the crosssection of $y=15 \mathrm{~mm}$, were used as inlet conditions for the current computational domain. The obtained spray jet flow field using RSM (Fig. 13) was compared with that in Fig. 10, which ensured the similar spray jet flow field due to the interpolation of inlet boundary conditions. 
For the analysis of simulation results, more attention was paid to the turbulent near wall flow on the spray target. Distributions of turbulent kinetic energy $k$ and the normal turbulent fluctuation $v^{\prime 2}$ in cells adjacent to the wall along the centre line of the spray pattern were depicted in Fig. 14. The differences between RSM and k- $\varepsilon$ model in the spray pattern centre are quite small, but higher $k$ and the normal turbulent fluctuation can be observed at $x= \pm 0.15 \mathrm{~m}$ for the results using the $k-\varepsilon$ model. However, it is interesting to examine the mean normal velocity component close to the wall. Figure 15 shows negative Uy component in wall cells by using RSM, whereas almost positive values with $\mathrm{k}-\varepsilon$ model. The negative Uy, in wall cells, that is in line with the spray jet direction will enhance the particle deposition.

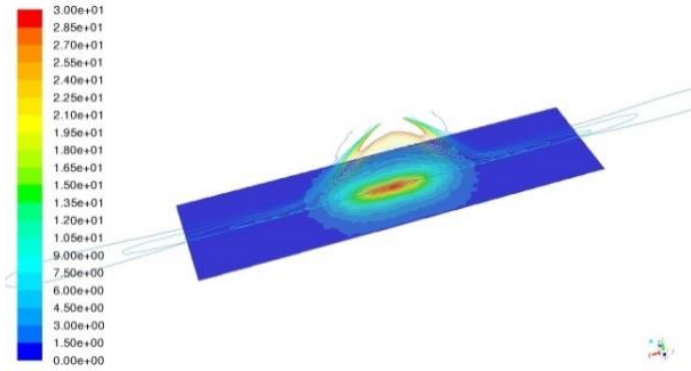

Figure 13. Calculated velocity contours colored by velocity magnitude $[\mathrm{m} / \mathrm{s}]$ in the cross-section $\mathrm{z}=0$ with hexahedral mesh model without atomizer. The static film thickness distribution on the plate is also overlaid.

Particle trajectory calculations were then carried out first using micro-sized particles (Fig. 3) to study the effect of turbulence model on particle deposition rate. The corresponding transfer efficiency TE $=64 \%$ for RSM and $63 \%$ for $\mathrm{k}-\varepsilon$ model were obtained.

The effect of $C_{L}$ on particle dispersion/deposition in EIM with RSM was also studied using $C_{L}=0.15$ and $C_{L}=0.8$. It was found that the influence of $C_{L}$ is quite small for high-inertia particles, i.e. large particles. However, the effect of $C_{L}$ for small particles cannot be neglected. Figure 16 shows sample trajectories of $0.5 \mu \mathrm{m}$ particle by using RSM and two values of $C_{L}$. With $C_{L}=0.15$ most of particles stream along the long axis of the elliptic spray pattern and away from two sides of the wall, which results in a quite dilute particle concentration in the direction of the short axis of the target. In contrast, a higher nanoparticle concentration was measured in the direction of the short target axis (Fig. 7) than that at the longer axis (Fig.8). The particle dispersion with $C_{L}=0.8$ in Fig. 16 shows similar trend as the experiment and is therefore considered to be reasonable. The TE with $C_{L}=0.15$ is $30 \%$ and $40 \%$ with $C_{L}=0.8$ in Fig. 16 .

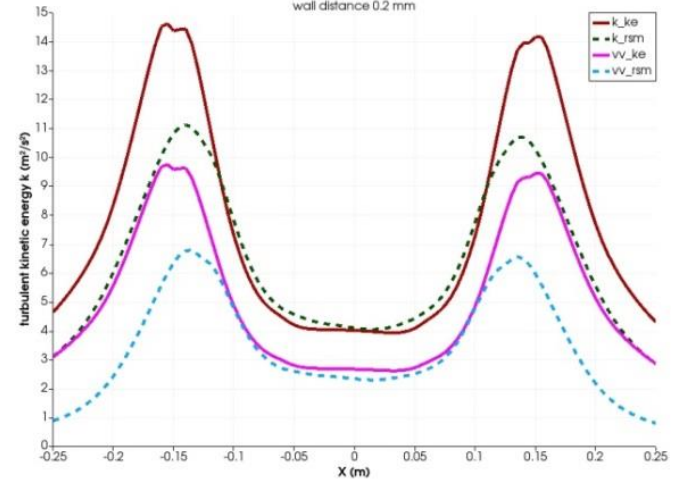

Figure 14. Turbulent kinetic energy and normal fluctuation $v^{\prime 2}$ in the wall cell
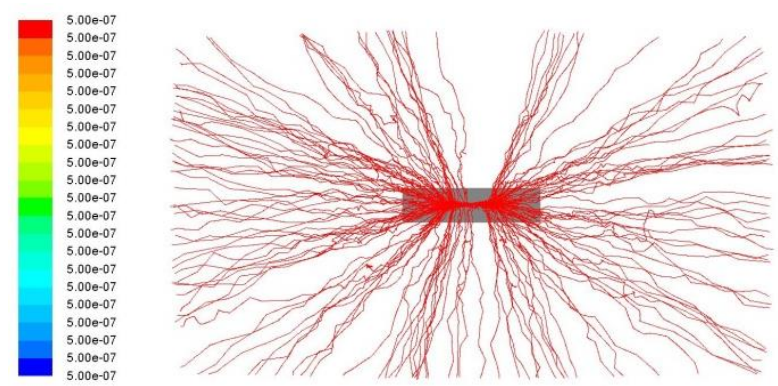

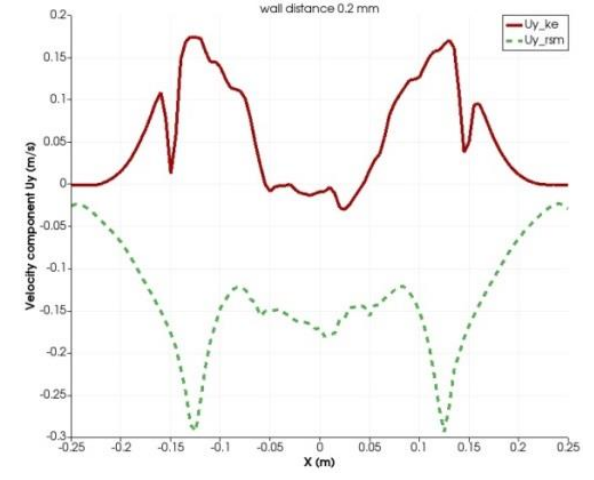

Figure 15. Normal velocity component Uy in the wall cell

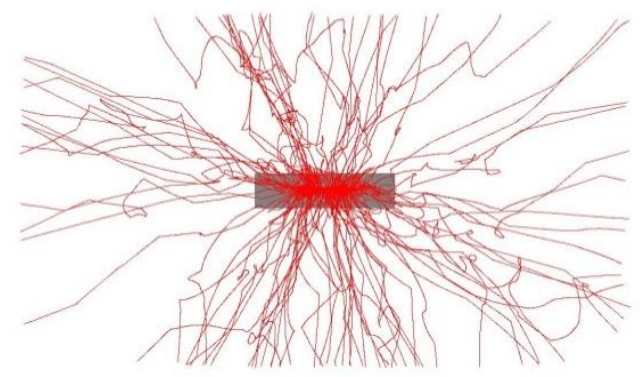

Figure 16. Trajectories of $0.5 \mu \mathrm{m}$ particles, left $C_{L}=0.15$, right $C_{L}=0.8$

Finally, the total nanoparticle distribution for the given operation conditions was fitted by means of the measured aerosol concentrations. The simulated concentrations of small paint aerosol particles were compared with the experiment at the sampling position $(\mathrm{L} 50 \mathrm{~cm}, \mathrm{~W} 0, \mathrm{H} 79.5 \mathrm{~cm})$ and shown in Fig. 17. In the figure, the experimental data was converted to obtain non-normalized particle concentrations, to be able to compare with those obtained from numerical simulations. For the simplification the particle distributions were regrouped and the background 
concentration was subtracted from the measured concentration. Based on the current experimental and numerical simulation results, the total number of nano and sub-micro particles in the range of diameter from $20-550 \mathrm{~nm}$ from the given spray gun is obtained, which is $1.56 \times 10^{7}$ per gram of paint ejection for water borne paint and $5.2 \times 10^{8}$ for clear coat. The results deliver important information for the further investigation of worker exposure to paint overspray.

\section{Conclusions}

Experimental and numerical studies of the determination of particle size distributions and concentrations in paint overspray created by a handcraft spray gun have been carried out. Although the droplet Sauter mean diameter obtained by micro particle size measurement is quite similar between two different paint materials, it was found that the solvent borne paint (clear coat) creates significantly higher number concentration of nano-sized droplets than the water borne paint. The relative low viscosity and strong solvent evaporation of clear coat could be the reason for the resulting atomisation behaviour. There were no significant differences of nano droplet concentrations between paints with and without manufactured nanomaterials, especially for the clear coat.

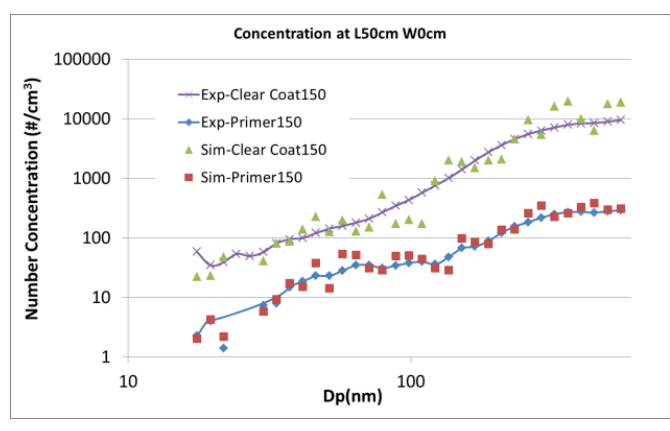

Figure 17. Comparison of simulated and measured nanoparticle concentrations at sampling position $\mathrm{L} 50 \mathrm{~cm}, \mathrm{~W} 0 \mathrm{~cm}$

Numerical simulations of droplet trajectories in spray booth both for micro and nano-sized droplets were carried out. The simulated two phase bulk flow field of a spay jet was validated by using measured film thickness distribution on the target. Particle dispersion and deposition for small-sized particles in turbulent near wall flow were then studied using two different turbulence models. The effect of turbulent time constant in the standard EIM in LPT was analysed. It was found that the default small value of $C_{L}$ yields unrealistic predictions for the sub-micro particle dispersion. Higher $C_{L}$ and RSM for near wall turbulent flow calculation were applied, which results in higher small-sized particle deposition than that using k- $\varepsilon$ model and standard EIM. Finally, representative nano and sub-micro particle distributions for a given spray gun were obtained based on the measured particle concentration in paint overspray, which delivers useful information for the further investigations of human exposure to a paint overspray in a practical paint booth and toxicological studies for evaluating the corresponding potential risk.

\section{Acknowledgements}

The present investigations have been supported by Verkehr, Innovation und Technologie, Austria (BMVIT) and Fraunhofer Society in Germany within a European joint research project NanoGeCo. This support is gratefully acknowledged by the authors.

\section{References}

[1] Kuhlbusch, T., Asbach C., Fissan, H., Göhler, D., and Stintz, M., Particle and Fibre Toxicology 2011, 8:22

[2] Carlton, G. N. \& Flynn, M. R. A, App. Occup. Environ. Hyg. 12, 375-382 (May 1997).

[3] Flynn, M. R. \& Sills, E. D., Annals of Occupational Hygiene 44, 191-202 (Aug. 2000).

[4] Ye, Q. and Domnick, J. Coat. Technol. Res., 14(2) 467-476 2017, DOI 10.1007/s11998-016-9867-4

[5] Ye, Q., Shen, B, Bauernhansl, T \& Dominick, J., Atomization and Sprays 25, 643-656 (2015).

[6] Ye, Q. \& Pulli, K., Coatings 2017, 7, 13.

[7] Göhler, D. \& Stintz, M., Journal of Nanoparticle Research 16, 1-15 (2014).

[8] Stintz, M, Technical Report, TU Dresden, 2016

[9] Wang, Y., \& James, P.W. (1999), International Journal of Multiphase Flow, 25, 551-558.

[10] Dehbi, A. (2008), International Journal of Multiphase Flow, 34, 819-828.

[11] Agnihotri, V., Ghorbaniasl, G., Verbanck, S., Lacor, C., J. of Aerosol Science 47 (2012) 39-47.

[12] Mehel, M., Sagot, B., Tanière, A., and Oesterlé, B., Int. J. Nonlinear Sci. Numer. Simul. 2012; 13(6): 417-425

[13] Stintz, M, Technical Report, TU Dresden, 2016

[14] Domnick, J. Gruseck, D., Pulli, K., Scheibe, A. Ye, Q., and Brinckmann, F., Chemical Engineering and Processing 50 (2011) 495-502

[15] Ye, Q., Tiedje, T. and Shen, B., ILASS - Europe 2014, 26th Annual Conference on Liquid Atomization and Spray Systems, 8-10 Sep. 2014, Bremen, Germany

[16] Graham, D.I., and James, P.W., IMA Journal of Mathematics Applied in Business \& Industry (1996) 7, 149-179 